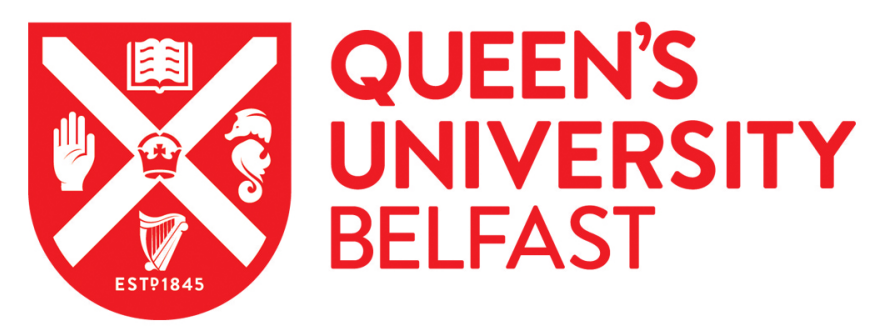

\title{
Anisotropy parameters in two-color two-photon above-threshold ionization
}

Hutchinson, S., Lysaght, M., \& Van Der Hart, H. W. (2013). Anisotropy parameters in two-color two-photon above-threshold ionization. Physical Review A (Atomic, Molecular, and Optical Physics), 88(2), [023424]. https://doi.org/10.1103/PhysRevA.88.023424

Published in:

Physical Review A (Atomic, Molecular, and Optical Physics)

Document Version:

Publisher's PDF, also known as Version of record

Queen's University Belfast - Research Portal:

Link to publication record in Queen's University Belfast Research Portal

Publisher rights

@2013 American Physical Society

\section{General rights}

Copyright for the publications made accessible via the Queen's University Belfast Research Portal is retained by the author(s) and / or other copyright owners and it is a condition of accessing these publications that users recognise and abide by the legal requirements associated with these rights.

Take down policy

The Research Portal is Queen's institutional repository that provides access to Queen's research output. Every effort has been made to ensure that content in the Research Portal does not infringe any person's rights, or applicable UK laws. If you discover content in the Research Portal that you believe breaches copyright or violates any law, please contact openaccess@qub.ac.uk. 
PHYSICAL REVIEW A 88, 023424 (2013)

\title{
Anisotropy parameters in two-color two-photon above-threshold ionization
}

\author{
Steven Hutchinson* \\ Department of Physics and Astronomy, University College London, Gower Street, London WC1E 6BT, United Kingdom \\ Michael A. Lysaght \\ Irish Centre for High End Computing, Tower Building, Trinity Technology and Enterprise Campus, Grand Canal Quay, Dublin 2, Ireland \\ Hugo W. van der Hart \\ Centre for Theoretical Atomic, Molecular and Optical Physics, School of Mathematics and Physics, \\ Queen's University Belfast, Belfast BT7 1NN, United Kingdom \\ (Received 9 July 2013; published 27 August 2013)
}

\begin{abstract}
We employ the time-dependent $R$-matrix (TDRM) method to calculate anisotropy parameters for positive and negative sidebands of selected harmonics generated by two-color two-photon above-threshold ionization of argon. We consider odd harmonics of an 800-nm field ranging from the 13th to 19th harmonic, overlapped by a fundamental $800-\mathrm{nm}$ IR field. The anisotropy parameters obtained using the TDRM method are compared with those obtained using a second-order perturbation theory with a model potential approach and a soft photon approximation approach. Where available, a comparison is also made to published experimental results. All three theoretical approaches provide similar values for anisotropy parameters. The TDRM approach obtains values that are closest to published experimental values. At high photon energies, the differences between each of the theoretical methods become less significant.
\end{abstract}

DOI: 10.1103/PhysRevA.88.023424

PACS number(s): $32.80 . \mathrm{Fb}, 32.80 . \mathrm{Rm}, 31.15 . \mathrm{A}-$

\section{INTRODUCTION}

In recent years, one of the most prominent areas of atomic physics has been the study of atomic processes on an ultrafast time scale [1]. This work has been driven by the development of ultrafast light sources capable of producing light pules with a duration in the attosecond region [2], which has enabled realtime experimental observation of ultrafast atomic behavior such as light-field-induced electron tunneling [3], the decay of an inner-shell vacancy [4], and the motion of a valence electron [5], while attosecond electron wave-packet interferometry has revealed information about the ultrafast dynamics of electron wave packets [6]. The key to sustaining these developments in pulse generation and application has been the characterization of light pulses of such short duration.

The characterization of attosecond pulses has proven to be highly challenging, as light pulses on the attosecond time scale have relatively low intensity. This renders most standard short-pulse characterization techniques impossible, since these tend to be based on intensity autocorrelation in nonlinear processes [7]. For example, techniques using nonlinear crystals such as frequency-resolved optical gating (FROG) [8] cannot be used (directly) since these crystals are highly absorbent in the XUV range. This has led to the development of new metrology methods which use the nonlinearity of processes such as high harmonic generation and multicolor two-photon ionization for attosecond pulse characterization. One of the most successful of these has been the reconstruction of attosecond beating by interference of two-photon transition (RABBITT) [9]. This method uses three-color two-photon ionization to generate two consecutive high harmonic signals and three associated sidebands. By observing the modulation

\footnotetext{
*steven.hutchinson@ucl.ac.uk
}

of the central common sideband relative to the time at which an IR field is applied, it is possible to determine the relative phase of each of the original harmonic pulses. Once the relative phase of all components has been obtained, the original pulse can be reconstructed [10].

Due to the importance of ATI sidebands for pulse characterization, there has been recent experimental interest in photoelectron angular distributions of individual ATI sidebands in two-color two-photon ionization processes. In particular, experimental measurements have recently been made to determine the photoelectron angular distributions of positive and negative sidebands of the 13th and 15th harmonics of argon in two-color two-photon above-threshold ionization [11]. In this study, experimental measurements were used to determine the anisotropy parameters of each sideband and the ratio between cross sections for positive and negative sidebands for each harmonic. A comparison was then made between experimental values and the values obtained from theoretical approaches using second-order perturbation theory with a model potential (MP) [12] and the soft photon approximation (SPA) [13]. Although there was reasonable agreement with experimental values, both of the theoretical models produced anisotropy parameters outside of the range of experimental error.

The discrepancy between experimental and theoretical results displayed in [11] creates an interest to apply other theoretical methods which account for more of the atomic structure to this type of problem. Both the model potential and the soft photon approximation methods are based on the single active electron (SAE) approximation. This approximation significantly reduces the computational complexity of modeling harmonic generation but cannot describe the full physics involved which may play a role on ultrashort time scales. Central to the MP approach [12] is the use of a model potential in order attempt to compensate for electron exchange and correlation effects which may provide a significant limitation 
on the accuracy of this approach. It is therefore of interest to investigate how sensitive these asymmetry parameters are with respect to the potential, by comparing with results from, for example, an ab initio approach.

Theoretical approaches that numerically solve the fulldimensional time-dependent Schrödinger equation for multielectron systems in a laser field with exact potentials acting on each of the electrons are available [14], however the complexity of such a system means that the problem is intractable for targets with more than two active electrons. The recently developed time-dependent $R$-matrix (TDRM) theory [15], however, provides a technique capable of describing the time-dependent response of a general multielectron system interacting with a laser field while employing $R$-matrix methods such as space partition to reduce the computational complexity. At the moment this method is limited to the emission of a single electron. Unlike the MP and SPA methods, the TDRM method uses the real potential acting on each electron as multielectron effects such as electron correlation and exchange are properly accounted for.

The TDRM method has already proven highly successful in applications to the study of ultrafast electron dynamics [16-18]. As sidebands in two-color two-photon abovethreshold ionization are of significant experimental [11] and theoretical [19] interest, with previous comparable work having already been performed [11], it is of interest to apply the TDRM method to two-color two-photon above-threshold ionization of argon. We use the same laser frequencies from this experiment to calculate anisotropy parameters for positive and negative sidebands, and the ratio between the cross sections of these sidebands, of the 13th and 15th harmonics using the TDRM method to enable a comparison with experimentally measured values. We also compare the previously applied MP [12] and SPA [13] theoretical methods by verifying anisotropy parameters and cross-section ratios for relevant sidebands using these methods. Finally, we extend our results beyond those that have been experimentally measured to include the 17th and 19th harmonics using the TDRM method, and, where available, the SPA and MP methods.

\section{TIME-DEPENDENT $R$-MATRIX THEORY}

The time-dependent $R$-matrix theory used throughout this study is an extension of standard $R$-matrix techniques for scattering processes to time-dependent processes. A thorough overview of this theory has been published previously [15], thus only a brief description is given here.

The TDRM method solves the time-dependent Schrödinger equation for a general $(N+1)$ electron atom or ion interacting with a laser pulse by employing the unitary form of the time evolution operator to rewrite the TDSE in the form of a CrankNicolson scheme as follows:

$$
\left[H\left(t_{q+1 / 2}\right)-E\right] \Psi\left(\mathbf{X}_{N+1}, t_{q+1}\right)=\Theta\left(\mathbf{X}_{N+1}, t_{q}\right),
$$

where

$$
\Theta\left(\mathbf{X}_{N+1}, t_{q}\right)=-\left[H\left(t_{q+1 / 2}\right)+E\right] \Psi\left(\mathbf{X}_{N+1}, t_{q}\right) .
$$

In Eqs. (1) and (2), $\mathbf{X}_{N+1}=\mathbf{x}_{1}, \mathbf{x}_{2}, \ldots, \mathbf{x}_{N+1}$ where $\mathbf{x}_{i} \equiv \mathbf{r}_{i} \sigma_{i}$ are the space and spin coordinates of the $i$ th electron. To implement the Crank-Nicolson scheme, we have introduced a discrete mesh in time with a discrete time step $\Delta t=$ $t_{q+1}-t_{q}$. The imaginary energy $E$ is then defined by this time step according to $E \equiv 2 i(\Delta t)^{-1} . H\left(t_{q+1 / 2}\right)$ represents the time-dependent Hamiltonian at the midpoint of times $t_{q}$ and $t_{q+1}$. We assume that the light field is spatially homogeneous and linearly polarized throughout. Following the analysis presented in earlier work [20], which demonstrated that the optimum choice of gauge for this type of problem was the length gauge, the length gauge is used to describe the laser interaction throughout.

To solve Eq. (1), we employ standard $R$-matrix techniques by partitioning the configuration space into two distinct regions: an internal region and an external region. The internal region is defined as a small region with radius $r=a_{\text {in }}$ chosen to enclose the core of the target, with all $(N+1)$ electrons contained within this region. In the internal region exchange and correlation effects are considered between all of the $(N+1)$ electrons. The external region is defined as a large spatial region $a_{\text {in }} \leqslant r \leqslant a_{\text {out }}$ where only the ejected $(N+1)$ th electron is present. The residual $N$ electrons are still considered, however they are confined to the internal region spatially with correlation effects accounted for by long-range potential matrices. Exchange effects between the ejected electron and the residual $N$ electrons are considered negligible and thus not included. The external region is chosen with $a_{\text {out }}$ large enough that the ejected electron wave function does not reach this boundary within the finite time considered. For computational reasons the external region is further subdivided into subregions of identical length.

In the internal region we expand the wave function $\Psi\left(\mathbf{X}_{N+1}, t_{q+1}\right)$ in an antisymmetric $R$-matrix basis $\psi_{k}$. To ensure Hermicity at the boundary $r=a_{\mathrm{in}}$, and to account for the component of the wave function that leaves the internal region box, we introduce a Bloch operator $\mathcal{L}$ which allows us to rewrite Eq. (1) in the form

$$
(H+\mathcal{L}-E) \Psi_{q+1}=\mathcal{L} \Psi_{q+1}+\Theta_{q},
$$

which has the formal solution

$$
\Psi=(H+\mathcal{L}-E)^{-1} \mathcal{L} \Psi+(H+\mathcal{L}-E)^{-1} \Theta .
$$

Solutions of this equation are found by expressing the wave function $\Psi$ in the inner region in terms of inner region eigenfunctions $\psi_{k}$ of the operator $(H+\mathcal{L})$ :

$$
\Psi\left(\mathbf{X}_{N+1}, t_{q+1}\right)=\sum_{k} \psi_{k}\left(\mathbf{X}_{N+1}\right) B_{k}\left(E, t_{q+1}\right),
$$

where $B_{k}$ are time-dependent expansion coefficients. To connect the internal region and the external region, we first consider the behavior of the internal region wave function at the boundary between regions. To this end, we project Eq. (4) onto the $n$ time-independent channel functions $\bar{\Phi}_{p}^{\gamma}$, which are formed by coupling the residual ion state $\Phi$ with the angular and spin functions of the continuum electron. By evaluating the resulting expression on the boundary $r=a_{\text {in }}$ we obtain the following expression:

$$
\mathbf{F}\left(a_{\text {in }}\right)=\mathbf{R} a_{\text {in }} \overline{\mathbf{F}}\left(a_{\text {in }}\right)+\mathbf{T}\left(a_{\text {in }}\right),
$$

where $\mathbf{F}$ is the reduced radial wave function and $\overline{\mathbf{F}}$ its first derivative. The terms $\mathbf{R}$ and $\mathbf{T}$ represent the $R$ matrix and $T$ vector, respectively. Formal definitions for each of these 
terms are available in Ref. [15]. The right-hand side of Eq. (6) consists of two main components. The $T$ vector arises from the action of the operator $(H+\mathcal{L}-E)^{-1}$ on the inhomogeneous term $\Theta$ in Eq. (4) and provides information about the flow of the wave function at $t=t_{q}$ through the boundary. The $\mathbf{R} a_{\text {in }} \overline{\mathbf{F}}\left(a_{\text {in }}\right)$ term is a correction to account for the components of the wave function that leave or enter the internal region, and thus provides information about the rate of flow of the unknown wave function at $t=t_{q+1}$ through the boundary. This term arises from the action of the Bloch operator in Eq. (4). By obtaining the vector $\mathbf{F}$ we may determine the expansion coefficients $B_{k}$ in Eq. (5) and consequently the full wave function $\Psi$ in the internal region at $t=t_{q+1}$. However, we must first determine the modified derivative functions $\overline{\mathbf{F}}$ from analysis of the external region.

In the external region we expand the wave function according to

$$
\Psi\left(\mathbf{X}_{N+1}, t_{q+1}\right)=\sum_{p=1}^{n} \bar{\Phi}_{p}^{\gamma}\left(\mathbf{X}_{N} ; \hat{\mathbf{r}}_{N+1}\right) r_{N+1}^{-1} F_{p}\left(r_{N+1}\right),
$$

where the reduced radial functions $F_{p}$ are analytic continuations of the functions defined on the internal region boundary in Eq. (6). As in the internal region, we introduce a Bloch operator to ensure Hermicity on the boundaries of each of the subregions in the external region. The formal solution to Eq. (1) in the external region then has the same form as Eq. (4). The expansion (7) is such that, by using similar techniques to those used in the internal region, we may then demonstrate that Eq. (6) is valid for any boundary between subregions in the external region.

Having validated Eq. (6) for any boundary, we may now develop an approach to determine the wave function $\mathbf{F}$ and its derivative $\overline{\mathbf{F}}$ in the external region. We propagate the $R$ matrix and $T$ vector outwards across the boundaries of each of the external region sectors through the use of Green's functions derived from the Hamiltonian, using the $R$ matrix and $T$ vector calculated on the internal region boundary $r=a_{\text {in }}$ from analysis of the internal region as the initial values. The details of the propagators used to accomplish this are provided in [15]. By choosing the external region outer boundary large enough to ensure that the wave function does not reach the outer limit $r=a_{\text {out }}$ in the time frame considered, we may impose the boundary condition $\mathbf{F}=0$ at $r=a_{\text {out }}$ for every time step. Using the $R$ matrix and $T$ vectors on the boundary of each subregion, we may then propagate this $F$ vector inwards to determine its values at every boundary, providing the wave function $\Psi$ at every point of the external region. Finally, we may also determine $\overline{\mathbf{F}}$ on the internal region boundary, and consequently the wave function $\Psi$ at $t=t_{q+1}$ in the internal region. Having now obtained the wave function for the entire configuration space at $t=t_{q+1}$, we may use this wave function as the starting point for the next iteration of the procedure.

To describe argon we use the $R$-matrix basis developed for single-photon ionization of $\mathrm{Ar}$ [21], which includes the $3 s^{2} 3 p^{5}{ }^{2} P^{o}$ and $3 s 3 p^{6}{ }^{2} S^{e}$ states of $\mathrm{Ar}^{+}$as target states, with all $3 s^{2} 3 p^{5} \epsilon l$ and $3 s 3 p^{6} \epsilon l$ channels with angular momentum up to an including $L_{\max }=19$ included in the description of argon. The internal region is chosen to extend to a radius of 20 a.u., with the set of continuum orbitals containing 70 continuum functions for each available angular momentum of the continuum electron. The external region is chosen to extend to a distance of 1826 a.u. and is composed of subsectors of width 2 a.u. which contain $40 \mathrm{~B}$ splines per channel with order $k=11$.

We consider irradiation by an EUV laser pulse overlapped by an IR dressing field. The IR laser pulse has a wavelength of $800 \mathrm{~nm}$ with a peak intensity of $5 \times 10^{10} \mathrm{~W} \mathrm{~cm}^{-2}$ and a pulse profile consisting of a three-cycle $\sin ^{2}$ ramp on, followed by two cycles at peak intensity and a three-cycle $\sin ^{2}$ ramp off. The EUV laser pulse corresponds to a selected harmonic of an $800-n m$ pulse ranging from the 13th to 19th harmonic and has a peak intensity $5 \times 10^{11} \mathrm{~W} \mathrm{~cm}^{-2}$. The EUV pulse for the $n$th harmonic is described by a $3 n$-cycle $\sin ^{2}$ ramp on, followed by $2 n$ cycles at peak intensity and a $3 n$-cycle $\sin ^{2}$ ramp off. The EUV and IR pulses start concurrently and are in phase.

\section{RESULTS}

We apply the TDRM method to calculate the wave function of the ejected electron when neutral argon is simultaneously irradiated by an EUV pulse corresponding to a selected harmonic of an 800-nm pulse ranging from the 13th to the 19th harmonic with intensity $5 \times 10^{11} \mathrm{~W} \mathrm{~cm}^{-2}$, overlapped by an IR dressing field with wavelength $800 \mathrm{~nm}$ and intensity $5 \times 10^{10} \mathrm{~W} \mathrm{~cm}{ }^{-2}$. The overlapping laser fields generate positive and negative sidebands of a central single-photon ionization peak. After the pulses end, the wave function is propagated for a further $1.87 \mathrm{fs}$.

In order to obtain the photoelectron angular distribution for the positive and negative sidebands generated in argon by a selected harmonic of the $800-\mathrm{nm}$ pulse and the overlapping fundamental IR pulse, we transform the wave function of the outer electron $1.87 \mathrm{fs}$ after the end of the laser pulse to obtain a two-dimensional (2D) momentum distribution for the ejected electron as explained previously [22]. This transformation assumes that the radial potential has become negligible in the region of the transformation. A typical photoelectron energy spectrum, obtained for the 15 th harmonic of the $800-\mathrm{nm}$ pulse, for the angle $\theta=0$ using the TDRM method is shown in Fig. 1. When compared to the experimentally measured photoelectron energy spectra provided in [11], we observe that the central harmonic peak is much narrower with the positive and negative sidebands clearly separated from this main peak. We also note

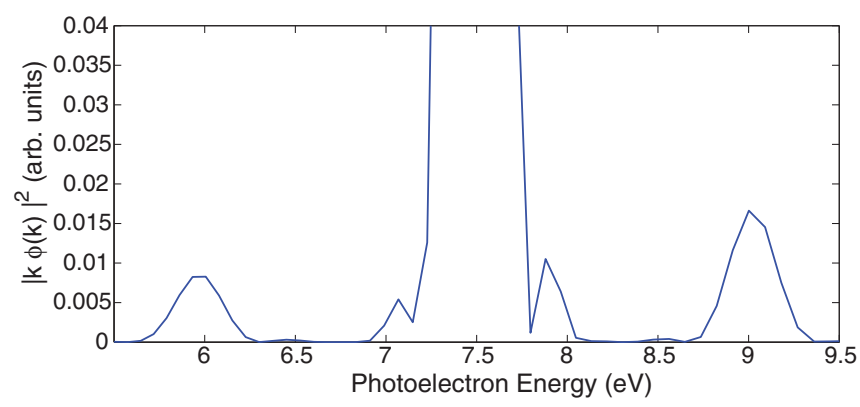

FIG. 1. (Color online) Photoelectron energy spectrum along the $y$ axis for the 15th harmonic and overlapping fundamental of the 800-nm pulse for argon showing the central single-photon ionization peak and associated positive and negative sidebands. 
that the intensity of the sidebands is significantly lower relative to the central harmonic peak than observed in the experimental results. This may be due to the shorter duration of the current pulses compared to the experimental pulse, as the central peak corresponds to a single-photon process, and as such has an intensity that scales linearly with pulse length, whereas the sidebands correspond to a two-photon process with an intensity that scales quadratically with pulse length. This use of a shorter pulse was imposed by computational limits. The much narrower harmonic peak and distinct sidebands allow us to safely integrate over the width of each sideband at a given angle to calculate the relevant photoelectron angular distribution $I(\theta)$ for each sideband.

As the laser fields considered in these calculations are both linearly polarized in the $z$ direction, and the target is unaligned, the photoelectron angular distributions for twophoton ionization are known to be of the form [23]

$$
I(\theta) \propto \frac{\sigma}{4 \pi}\left[1+\beta_{2} P_{2}(\cos \theta)+\beta_{4} P_{4}(\cos \theta)\right],
$$

where $\beta_{n}$ are the normalized anisotropy parameters. Having calculated the photoelectron angular distributions $I(\theta)$ for sidebands of high harmonics of argon using the TDRM method, we fit Legendre polynomials of the form of Eq. (8) to the angular distribution to obtain the normalized anisotropy parameters $\beta_{n}$. The ratio $\sigma^{(+)} / \sigma^{(-)}$between the positive and negative sidebands is obtained by comparing the constant scaling factor when fitting the Legendre polynomials to each of the sidebands. This ratio should thus be considered a ratio of electron emission yields rather than a ratio of cross sections. Anisotropy parameters for the SPA and MP methods have been verified using the same technique, using data generated by the TDRM approach for single-photon ionization and the tabulated data [12], respectively.

For odd numbered harmonics ranging from the 13th to 19th harmonic of the 800-nm pulse, Table I presents anisotropy parameters for positive and negative sidebands and crosssection ratios using the MP, SPA, and TDRM methods where available. Also provided for the 13th and 15th harmonics are experimentally measured anisotropy parameters from [11]. In Table I anisotropy parameters for negative sidebands are denoted by superscript $(-)$, and likewise positive sidebands are denoted by superscript $(+)$. The experimental data in [11] were compared with results from MP [12] and SPA [13]. We have therefore included anisotropy parameters obtained via these approaches as well.

The anisotropy parameters and cross-section ratios calculated using the TDRM method show that for each of the selected harmonics the negative sideband is smaller in magnitude than the positive sideband. This trend is observed in all of the results presented in Table I. We also note that as the energy of the harmonic increases the anisotropy parameters increase, but by diminishing amounts. This is demonstrated particularly clearly for the positive sideband, where values for the 17th and 19th harmonic are highly similar. Significantly, when using the TDRM method, sidebands with the same energy have differing anisotropy parameters for positive and negative sidebands, with negative sidebands having lower values than a positive sideband at the same energy. This demonstrates that there are slight differences in the physics of emission and absorption processes involving the IR photon.

The values we obtain for the anisotropy parameters using the TDRM method in Table I demonstrate varying agreement with experimental values. Qualitatively, the asymmetry parameters behave in a similar fashion in experiment and theory. The quantitative agreement between TDRM and experiment is best for positive sidebands with the TDRM method providing values for the anisotropy parameters of the positive sideband of the 13th high harmonic that lie entirely within the experimental range of values. There remains a discrepancy however between anisotropy parameters measured experimentally and those obtained using the TDRM method for the negative sideband. For example the $\beta_{2}^{(-)}$parameter for the 15th harmonic calculated using the TDRM method lies well outside the range of experimental values. This may

TABLE I. Anisotropy parameters and cross-section ratios for sidebands generated by the 13th to 19th harmonics of the 800-nm pulse overlapped by the fundamental IR pulse in argon.

\begin{tabular}{|c|c|c|c|c|c|}
\hline & $\sigma^{(+)} / \sigma^{(-)}$ & $\beta_{2}^{(-)}$ & $\beta_{4}^{(-)}$ & $\beta_{2}^{(+)}$ & $\beta_{4}^{(+)}$ \\
\hline 13th HH experiment & $1.39 \pm 0.05$ & $1.52 \pm 0.05$ & $0.21 \pm 0.05$ & $2.37 \pm 0.10$ & $0.63 \pm 0.04$ \\
\hline 13th HH MP & 1.42 & 2.08 & 0.50 & 2.72 & 1.18 \\
\hline 13th HH SPA (TDRM $\beta$ ) & 1.75 & 2.32 & 0.43 & 2.52 & 0.70 \\
\hline 13th HH TDRM & 1.53 & 1.87 & 0.28 & 2.32 & 0.67 \\
\hline 15th HH experiment & $1.27 \pm 0.05$ & $1.63 \pm 0.12$ & $0.54 \pm 0.15$ & $2.48 \pm 0.06$ & $0.69 \pm 0.05$ \\
\hline 15th HH MP & 1.28 & 2.42 & 0.72 & 2.87 & 1.27 \\
\hline 15th HH SPA (TDRM $\beta$ ) & 1.31 & 2.52 & 0.70 & 2.63 & 0.84 \\
\hline 15th HH TDRM & 1.35 & 2.25 & 0.52 & 2.58 & 0.88 \\
\hline \multicolumn{6}{|l|}{ 17th HH experiment } \\
\hline 17th HH MP & 1.22 & 2.57 & 0.84 & 2.92 & 1.28 \\
\hline 17th HH SPA (TDRM $\beta$ ) & 1.10 & 2.63 & 0.84 & 2.69 & 0.92 \\
\hline 17th HH TDRM & 1.13 & 2.36 & 0.63 & 2.72 & 1.06 \\
\hline \multicolumn{6}{|l|}{ 19th HH experiment } \\
\hline 19th HH MP & & 2.64 & 0.91 & & \\
\hline 19th HH SPA (TDRM $\beta$ ) & & 2.69 & 0.92 & & \\
\hline 19th HH TDRM & 1.16 & 2.49 & 0.74 & 2.76 & 1.05 \\
\hline
\end{tabular}


be explained in part by differences in the experimental and theoretical pulse profiles. The experimental photoelectron angular distributions were obtained through subtracting the background single-photon ionization (SPI) harmonic peaks, however the experimental pulse is much broader than the idealized theoretical pulse. The experimental intensity profile of the 13th and 15th SPI harmonic peaks of argon in [11] show that there is a greater background signal present in the region of the negative sidebands than the positive sidebands of each of these harmonics. This may affect the extent to which theory and experiment can be compared. The narrow theoretical pulse ensures that background subtraction is not necessary for the TDRM method.

For the MP calculations, we obtain a photoelectron angular distribution for each of the relevant sidebands using the technique presented in [12], before fitting Legendre polynomials in the form of Eq. (8). As data for the positive sideband of the 19th harmonic was not provided in [12], these values are omitted in Table I. The MP method is limited to considering a single active electron in the configuration space, thus limiting its ability to describe multielectron effects such as correlation properly. The MP method accounts for correlation and exchange effects through the use of a modified potential chosen to reproduce the eigenenergies and binding energies of the singly excited states of argon. It therefore excludes, for example, effects from the $3 s^{3} p^{6} \epsilon l$ channels. In order to investigate how appropriate this potential is for quantitative studies, it is useful to investigate how it compares to one that represents the Ar atom from first principles.

When compared with the anisotropy parameters obtained using the TDRM method, the MP approach provides values for the anisotropy parameters that are in general agreement with those obtained in the TDRM method. However, it can also be seen that the MP anisotropy parameters are consistently higher than those obtained using TDRM: the $\beta_{2}^{(-)}$and $\beta_{4}^{(-)}$parameters are approximately 0.2 higher than those calculated using the TDRM approach, and similarly the $\beta_{2}^{(+)}$and $\beta_{4}^{(+)}$are between 0.2-0.5 higher than the TDRM equivalent. Since the TDRM asymmetry parameters are in all cases closer to experiment than the MP parameters, it appears that the potential used in the TDRM calculations provides the better approximation to the true Ar potential.

The second approach that we compare with is the softphoton approximation. In this approximation, the expression for the photoelectron angular distributions for two-color above-threshold ionization is given as [13]

$$
\left(\frac{d \sigma^{(n)}}{d \theta}\right)_{E_{k}}=\frac{k}{k_{0}} J_{n}^{2}\left(\alpha_{\mathbf{0}} \cdot \mathbf{K}\right)\left(\frac{d \sigma^{(0)}}{d \theta}\right),
$$

where $n$ is defined by the number of low-energy photons exchanged after absorbing a single high-energy photon, with the sign of $n$ determined by emission $(n>0)$ or absorption $(n<0)$ of the low-energy IR photon. $n$ thus corresponds to the sideband in question (positive sidebands are a result of absorption and negative sidebands are a result of stimulated emission). $d \sigma^{(0)} / d \theta$ indicates the differential cross section for single-photon ionization given at the sideband energy $E_{k}$. The $\alpha_{0}$ term represents the classical excursion vector of a free electron in a laser field, while $\mathbf{K}$ is the momentum
TABLE II. Single-photon anisotropy parameters and photoionization cross sections calculated using the TDRM method, and compared to the values used in [11]. The latter data were originally obtained from [24] and [25], respectively.

\begin{tabular}{lcccc}
\hline \hline Harmonic & $\begin{array}{c}\text { TDRM } \\
\beta_{2}^{(0)}\end{array}$ & $\begin{array}{c}\text { TDRM } \\
\sigma(\mathrm{Mb})\end{array}$ & $\begin{array}{l}{[11]} \\
\beta_{2}^{(0)}\end{array}$ & $\begin{array}{c}{[11]} \\
\sigma(\mathrm{Mb})\end{array}$ \\
\hline 12 & 0.4951 & 32.78 & 0.4 & 37.6 \\
14 & 0.9297 & 34.34 & 1.1 & 37.0 \\
16 & 1.2040 & 33.64 & 1.4 & 34.9 \\
18 & 1.3975 & 30.37 & & \\
\hline \hline
\end{tabular}

transfer between the electron's incoming wave vector and its final state wave vector. $J_{n}$ represents a Bessel function. At low intensity, following [11], the assumption is made that $n= \pm 1$ sidebands dominate and $\left|\alpha_{0} \cdot K\right| \ll 1$. Consequently the Bessel functions $J_{ \pm 1}^{2}(z)$ are proportional to $\cos ^{2} \theta$, which results in the sideband angular distributions given by Eq. (9) with $n= \pm 1$ being determined primarily by $\cos ^{2} \theta$ times the single-photon differential cross sections. These single-photon differential cross sections behave as a function of $\theta$ as [13]

$$
I(\theta) \propto 1+\beta_{2} P_{2}(\cos \theta) .
$$

We therefore obtain photoelectron angular distributions determined by the single-photon anisotropy parameter $\beta_{2}^{(0)}$. The value of $\beta_{2}^{(0)}$ is chosen to correspond to the energy of each sideband. To enable a comparison between the TDRM and SPA methods in two-color two-photon ATI, we choose to use $\beta_{2}^{(0)}$ parameters calculated using the TDRM method as our input for the SPA method. This allows us to investigate of the differences between the soft-photon approximation and the TDRM approach with the least influence from differences in the absorption of the harmonic photon. The values of $\beta_{2}^{(0)}$ are shown in Table II. When considering the SPA results, we note that the values we obtained differ from those used in [11] where experimental values for $\beta_{2}$ were chosen.

This protocol for the SPA method has several consequences for the angular distributions. First of all, the choice of the single-photon asymmetry parameter at the final-state energy means that the method predicts no difference between the asymmetry parameters for the $N-1$ harmonic + IR absorption, and the $N+1$ harmonic + IR emission. This behavior is seen in Table I. A second consequence is that the SPA predicts a zero in the angular distribution perpendicular to the polarization direction of the laser fields, so that

$$
\beta_{4}=\frac{4}{3}\left(\beta_{2}-2\right),
$$

as can also be seen from Table I.

Compared with the TDRM results, the anisotropy parameters for the SPA approach in Table I are highly similar for positive sidebands with agreement between the two approaches improving with higher energy harmonics. For negative sidebands however, the SPA approach provides anisotropy parameters that are consistently higher than those obtained using TDRM ranging from 0.20 for the $\beta_{2}^{(-)}$parameter of the 19th harmonic to 0.45 for the $\beta_{2}^{(-)}$parameter of the 13th harmonic. The effect of the atomic potential is therefore 
significantly more apparent for the negative sideband than for the positive sideband. Compared with experimental results the SPA approach is similar to the TDRM approach for the positive sideband of the 15 th harmonic, and only marginally worse for the positive sideband of the 13th harmonic. For negative sidebands the TDRM approach provided better approximations to the experimental results than the SPA approach, although neither method lies entirely within the experimental error bars. All of the values obtained using the SPA method lie outside the range of experimental error.

The overall comparison between the different approaches shows that the TDRM method provides anisotropy parameters with a slightly better agreement with experiment than either the SPA or MP methods. The discrepancy between the TDRM approach and the SPA and MP methods is demonstrated most notably for negative sidebands of the 13th and 15th harmonics. It is not surprising that the main differences are seen for these sidebands, since the atomic potential affects electron motion more for the lower harmonics than for higher harmonics. Threshold effects also will be more influential for lower harmonics. As the order of the harmonic is increased, the SPA and MP methods produce increasingly similar anisotropy parameters. However, they remain higher than the TDRM parameters. The comparison of TDRM and SPA parameters is most sensitive to the description of the continuum wave functions, since the ionization stage of the process is described by the TDRM method in both cases. The comparison with the MP method, on the other hand, is significantly affected by the differences in the effective potentials in the two methods during the ionization stage as well. This increases the potential for deviation between the methods. This may account for the generally better agreement between the TDRM and the SPA approach than between TDRM and MP.

The ratios between cross sections for positive and negative sidebands presented in Table I indicate the relative strength of the absorption and emission of an IR photon (corresponding to a positive and negative sideband, respectively) for a given harmonic. The ratios obtained using the TDRM method demonstrate that absorption process of an IR photon is the more likely process at all considered harmonics. However, the sidebands tend towards equality at higher harmonics. These ratios obtained using the TDRM method are larger than the experimentally obtained ratio by about $7-10 \%$, and lie outside the range of experimental error. As indicated earlier, this may in part be due to differences between the theoretical model of the harmonic radiation and the actual experimental frequency profile.

The cross-section ratios predicted by the MP method for the 13th and 15th harmonics are in excellent agreement with experimental ratios, and slightly lower than those obtained using TDRM. The MP values display the same general downward trend with increasing energy as the TDRM values, however for the 17 th harmonic the ratio predicted by the MP method is higher than both the SPA and TDRM approaches. Despite strong agreement with experimental values, there are noticeable differences in the asymmetry parameters, which suggests that the MP approach does not describe the twophoton ionization process in full detail.

Within the SPA approach, the ratio between the cross sections for a positive and negative sideband within the SPA is obtained by integrating Eq. (9) over all angles [11],

$$
\frac{\sigma^{(+)}}{\sigma^{(-)}} \propto \frac{k^{(+)}}{k^{(-)}} \frac{\sigma^{(0,+)}}{\sigma^{(0,-)}} \frac{\left[5+2 \beta_{2}^{(0)}\right]_{E_{k(+)}}}{\left[5+2 \beta_{2}^{(0)}\right]_{E_{k(-)}}},
$$

where $\beta_{2}^{(0)}$ is the single-photon anisotropy parameter from Eq. (10) at the energy $E_{k( \pm)}$ corresponding to the positive and negative sidebands for a selected harmonic, $k^{(+) /(-)}$indicates the momentum of the outgoing electron at the positive $(+)$ and negative $(-)$ sideband, and $\sigma^{(0)}$ indicates the photoionization cross section at the photon energy corresponding to the sideband energy. In order to determine this ratio, we have obtained single-photon ionization yields at photon energies corresponding to the sideband energies, and derived singlephoton ionization cross sections from them. These cross sections are reported in Table II.

The cross-section ratio obtained using the SPA method for the 13th harmonic is significantly larger than both the TDRM and MP methods at this energy. For higher harmonics, the SPA cross sections are found to be in very good agreement with the TDRM method. This suggests that, as was the case with anisotropy parameters, at lower energies the atomic potential and threshold effects, which are not included in the SPA method, have a significant effect on sideband generation process. At higher energies these processes are less significant, thus the SPA method predicts values that are largely similar to the TDRM method. The ratio for the 15th harmonic is in excellent agreement with experiment and both the MP and TDRM approaches.

\section{CONCLUSIONS}

We have applied the TDRM method to calculate the anisotropy parameters for positive and negative sidebands of the 13th to 19th harmonics of an 800-nm pulse overlapped by an IR dressing field in two-color two-photon abovethreshold ionization of argon and compared to those obtained experimentally, and by the MP and SPA methods. Overall, the asymmetry parameters obtained by all three theoretical methods are in general agreement with each other. At a detailed level, the anisotropy parameters calculated using the TDRM method are found to be generally smaller than those obtained using the SPA and MP approaches. Of the SPA and MP methods, the SPA approach provided anisotropy parameters closer to those predicted by TDRM method and measured experimentally for positive sidebands, but also produced values with the lowest degree of agreement with experiment for negative sidebands. The MP method provides values closer to TDRM and experiment than SPA for negative sidebands. For higher photon energies the differences between the TDRM method and the SPA and MP methods decrease.

The anisotropy parameters calculated using the TDRM method are in good agreement with those predicted experimentally and show an improvement in agreement with experimental results when compared to anisotropy parameters obtained using the MP and SPA methods. This demonstrates that the TDRM method gives some improvement in theoretical modeling of two-color two-photon above-threshold ionization of argon relative to the MP and SPA methods. This improvement probably originates from a more accurate description of the potential seen by the outer electron. While the TDRM 
method shows better agreement with experiment than either the SPA or MP methods, the anisotropy parameters obtained still remain mostly outside of the range of experimental values. Some of these differences may be due to differences between the frequency profile used in the theoretical calculations for the harmonic laser pulse and the experimental frequency profile.

\section{ACKNOWLEDGMENTS}

S.H. was supported by the Department of Employment and Learning NI under the Programme for Government. H.W.H. was supported by the UK Engineering and Physical Sciences Research Council under Grant No. G/055416/1.
[1] F. Krausz and M. Ivanov, Rev. Mod. Phys. 81, 163 (2009).

[2] R. Kienberger, E. Goulielmakis, M. Uiberacker, A. Baltuska, V. Yakovlev, F. Bammer, A. Scrinzi, T. Westerwalbesloh, U. Kleineberg, U. Heinzmann et al., Nature (London) 427, 817 (2004).

[3] M. Uiberacker, T. Uphues, M. Schultze, A. J. Verhoef, V. Yakovlev, M. F. Kling, J. Rauschenberger, N. M. Kabachnik, H. Schroder, M. Lezius et al., Nature (London) 446, 627 (2007).

[4] M. Drescher, M. Hentschel, R. Kienberger, M. Uiberacker, V. Yakovlev, A. Scrinzi, T. Westerwalbesloh, U. Kleineberg, U. Heinzmann, and F. Krausz, Nature (London) 419, 803 (2002).

[5] E. Goulielmakis, Z.-H. Loh, A. Wirth, R. Santra, N. Rohringer, V. S. Yakovlev, S. Zherebtsov, T. Pfeifer, A. M. Azzeer, M. F. Kling et al., Nature (London) 466, 739 (2010).

[6] T. Remetter, P. Johnsson, J. Mauritsson, K. Varju, Y. Ni, F. Lepine, E. Gustafsson, M. Kling, J. Khan, R. Lopez-Martens et al., Nat. Phys. 2, 323 (2006).

[7] I. A. Walmsley and C. Dorrer, Adv. Opt. Photon. 1, 308 (2009).

[8] R. Trebino, K. W. DeLong, D. N. Fittinghoff, J. N. Sweetser, M. A. Krumbügel, B. A. Richman, and D. J. Kane, Rev. Sci. Instrum. 68, 3277 (1997).

[9] P. M. Paul, E. S. Toma, P. Breger, G. Mullot, F. Aug, P. Balcou, H. G. Muller, and P. Agostini, Science 292, 1689 (2001).

[10] S. A. Aseyev, Y. Ni, L. J. Frasinski, H. G. Muller, and M. J. J. Vrakking, Phys. Rev. Lett. 91, 223902 (2003).
[11] L. H. Haber, B. Doughty, and S. R. Leone, J. Phys. Chem. A 113, 13152 (2009).

[12] E. S. Toma and H. G. Muller, J. Phys. B: At. Mol. Opt. Phys. 35, 3435 (2002).

[13] A. Maquet and R. Taïeb, J. Mod. Opt. 54, 1847 (2007).

[14] E. S. Smyth, J. S. Parker, and K. T. Taylor, Comput. Phys. Commun. 114, 1 (1998).

[15] M. A. Lysaght, H. W. van der Hart, and P. G. Burke, Phys. Rev. A 79, 053411 (2009).

[16] M. A. Lysaght, P. G. Burke, and H. W. van der Hart, Phys. Rev. Lett. 102, 193001 (2009).

[17] M. A. Lysaght, P. G. Burke, and H. W. van der Hart, Phys. Rev. Lett. 101, 253001 (2008).

[18] M. A. Lysaght, S. Hutchinson, and H. W. van der Hart, New J. Phys. 11, 093014 (2009).

[19] V. Véniard, R. Taïeb, and A. Maquet, Phys. Rev. A 54, 721 (1996).

[20] S. Hutchinson, M. A. Lysaght, and H. W. van der Hart, J. Phys. B: At. Mol. Opt. Phys. 43, 095603 (2010).

[21] P. G. Burke and K. T. Taylor, J. Phys. B: At. Mol. Phys. 8, 2620 (1975).

[22] H. W. van der Hart, M. A. Lysaght, and P. G. Burke, Phys. Rev. A 77, 065401 (2008).

[23] K. L. Reid, Annu. Rev. Phys. Chem. 54, 397 (2003).

[24] K. T. Taylor, J. Phys. B 10, L699 (1977).

[25] B. Henke, E. Gullikson, and J. Davis, At. Data Nucl. Data Tables 54, 181 (1993). 